\title{
Load balancing mechanisms for indoor temporarily overloaded heterogeneous femtocell networks
}

\author{
Alejandro Aguilar-Garcia*, Raquel Barco, Sergio Fortes and Pablo Muñoz
}

\begin{abstract}
Macrocell and small cell deployments and self-organizing network (SON) techniques work together to increase indoor cellular network capacity and ensure better quality of service (QoS). As a consequence of uneven local user densities and temporal or spatial fluctuations of traffic, the network may suffer overload situations, which partially degrades network performance. Load balancing self-optimization introduces automatic and intelligent mechanisms to tune network parameters in order to improve the overall cellular network performance. This paper proposes novel load balancing methods based on fuzzy logic controllers (FLC) that evaluate temporarily overloaded situations and resize cell coverage areas by an adaptive process of adjusting cell transmission power. To accomplish this goal, classical network indicators are analyzed (e.g., call blocking ratio, available radio resources) while a novel and simple, although powerful, indicator (not mentioned in the literature yet) is additionally proposed as the system input. This indicator is related to the maximum allowed number of users in a femtocell. The proposed methods have been evaluated and compared with the literature in a realistic scenario.
\end{abstract}

Keywords: Self-organizing networks; Self-optimization; Femtocell; Indoor; Load balancing; Fuzzy logic controller

\section{Introduction}

The requirements posed by the massive expansion of smartphones and tablets have led to heterogeneous cellular networks (HCNs), which comprise different radio access technologies (RAT) and several cell sizes (macrocell, picocell, or femtocell). The complexity in the operation of HCNs demands new network management techniques. In this sense, self-organizing networks (SON) have been identified by the Third Generation Partnership Project (3GPP) [1] and the Next Generation Mobile Networks (NGMN) [2] as a key feature to intelligently automate network management procedures in future networks [3,4].

Nowadays, most cellular traffic is generated indoors (e.g., home, work, shopping malls), where there is often a lack of coverage or insufficient quality of service (QoS). In these cases, network operators are offering femtocells to overcome the indoor issues, i.e., short-range, low-power, low-cost, and plug and play small cells usually designed to support $4,8,16$, or 32 users in connected mode [5]. In

\footnotetext{
* Correspondence: aag@ic.uma.es

Departamento de Ingeniería de Comunicaciones, Universidad de Málaga, Andalucía Tech, Campus de Teatinos s/n, 29071 Málaga, Spain
}

these scenarios, femtocells together with SON techniques can help, on the one hand, operators by reducing their capital and operational expenditure (CAPEX/OPEX) and, on the other hand, users by increasing the mobile phone battery lifetime and enhancing the user's QoS, due to the user-femtocell proximity.

Additionally, network traffic (voice and data) and local user densities present temporal and spatial variations. In these conditions, indoor environments might suffer from serious network problems because most traffic could be located in the same cell(s) during short periods. For example, people waiting for the bus/train, or any other public transport, may be connected to a particular cell, producing high levels of traffic and leading it to cellular network saturation. Another example is easily found in shopping malls, where a temporal spectacle or event could gather many people interested in taking pictures to share them in social networks. A simple solution to support these situations could be to plan the network to offer the maximum expected resources all the time. Nevertheless, this solution would largely increase operators' expenditures. 
In the literature, among SON techniques and focusing on self-optimization mechanisms, several works have been centered in the macrocell case. By contrast, indoor environments present hard and difficult conditions to manage the cellular network due to the cell overlapping, lack of coverage, interference, etc. This implies a challenge for researchers in the development of SON mechanisms [6-9]. In this sense, self-x techniques (i.e., autonomous mechanisms like self-configuration, self-optimization, and self-healing) in femtocell networks are currently a hot topic [10].

Regarding the area of self-optimization at indoor networks, mobility load balancing (MLB) use case defined by the 3GPP [3], aims to reallocate users from overloaded cells to low loaded neighboring cells in order to increase network capacity and improve the user's QoS. Load balancing mechanisms have been also widely studied in the literature at outdoor and indoor scenarios [11-14]. Those techniques tune network parameters to reach a better configuration that alleviates the congestion situation. In this context, focusing on indoor and femtocell environments, reference [15] presented a dynamic power control method for balancing data traffic based on radio propagation models and users' location, being a challenge for network operator to get the latter information. Simple and low-complexity methods based on fuzzy logic controllers (FLC) are proposed in [16], which investigated the problem of re-distributing traffic demand between long-term evolution (LTE) femtocells. This work also compared different traffic sharing techniques. A similar fuzzy logic approach is presented in [17], where cell transmission power and handover margins are tuned to solve persistent congestion problems in enterprise LTE environments, based on call blocking rate. A more computationally complex method [18] obtained better performance than previous studies through a fuzzy rule-based reinforcement learning system, while reference [19] proposed a distributed method to achieve automatic load balancing based on a flowing water method. However, none of these previous works focused on femtocell environments have taken into account the femtocell limitation in the number of simultaneous users which is an important restriction in this kind of base stations (macrocells or other type of small cells do not have such restriction). Moreover, these previous works were designed to solve localized and persistent congestion problems, disregarding the challenge related to temporary congestion issues.

The femtocell limitation in the maximum number of simultaneous users was taken into account in [20], where a handover algorithm based on the users' speed and QoS was proposed. That work evaluated whether a handover was necessary or not according to the previous metrics (speed and QoS), but the algorithm was not analyzed under overload situations.
In femtocells, restrictions in the maximum number of connected users is much severe than in macrocells, due to the femtocell processing and capacity limitations. To the best of the authors' knowledge, the use of the number of users in connected mode over the maximum number of simultaneous users in the femtocell as a key parameter of self-optimization mechanisms in indoor scenarios has not been considered in previous works (beyond the reference [20], whose focus was not load balancing). As this indicator is a powerful metric to be considered in femtocell scenarios, this paper analyzes some previous studies and proposes new and low complexity load balancing mechanisms based on the cell available bandwidth and/or the femtocell limitation in the number of simultaneous users by an adaptive cell resizing. Therefore, the contribution of this paper is minimizing the impact of temporal overloaded situations that degrade network performance based on the study of two network indicators (available bandwidth and cell capacity in terms of the number of users). In consequence, the main aim is to demonstrate the importance of including the information of 'the number of active users' in self-optimization procedures at indoor femtocell environments. A comparative summary of those algorithms is carried out in the evaluation section where their advantages/disadvantages are discussed.

This paper is organized as follows: The problem description is presented in Section 2. An explanation about fuzzy logic controllers is performed in Section 3. Section 4 describes the proposed load balancing methods. The performance analysis is studied in Section 5. Finally, the conclusions are drawn in Section 6.

\section{Problem description}

Occasional events or unpredictable situations could cause unexpected overload conditions in cellular networks, especially at indoor scenarios. These temporal variations, combined with the time-variant fading and coverage holes caused by reflections and obstacles, could negatively affect network performance.

The most suitable solution to reduce or avoid cellular congestion situations is by resizing the cell coverage areas. These kinds of load balancing algorithms set different radio parameters to adjust service areas. Consequently, the traffic is shared along the network by handing over those users served by a congested cell to the most suitable neighboring cell with spare resources.

These temporal network congestions must be evaluated from two perspectives. Firstly, from the users' side, the performance is assessed according to their experienced QoS. In particular, it is evaluated by measuring two network indicators which must be as low as possible: blocking ratio (BR) and outage ratio (OR). The $\mathrm{BR}$, the inverse of the network accessibility, is defined as the 
ratio of the number of blocked connections to the number of new users that attempt to access the network:

$$
\mathrm{BR}=\frac{N_{\text {blocked_users }}}{N_{\text {new_users }}}=\frac{N_{\text {blocked_users }}}{N_{\text {blocked_users }}+N_{\text {active_users }}}
$$

The other indicator, OR, is the complementary of the network retainability. More specifically, OR is described as the probability that an existing network connection is in standby mode before it is finished, i.e., it is the ratio of the number of standby connections to the total number of connections that are accepted by the network. The standby mode situation could be due either to a bad signal-to-interference-plus-noise ratio (SINR) of users $\left(\mathrm{OR}_{\mathrm{q}}\right)$ or a temporary lack of network resources $\left(\mathrm{OR}_{\mathrm{s}}\right)$ :

$$
\mathrm{OR}=\mathrm{OR}_{\mathrm{q}}+\mathrm{OR}_{\mathrm{s}}
$$

Secondly, from the operator point of view, another indicator must also be taken into account: the amount of signaling data. A key indicator to measure that information is the handover ratio (HOR), which reflects the average number of handovers per users, in consequence, the network signaling data. It is defined as:

$$
\mathrm{HOR}=\frac{N_{\text {handover }}}{N_{\text {active_users }}}
$$

where $N_{\text {handover }}$ is the number of handovers and $N_{\text {active_users }}$ is the number of active users in the network. Low values of HOR are desirable.

\section{Fuzzy logic controllers}

Several load balancing methods have been proposed in the literature, as described in Section 1. Focusing on indoor heterogeneous networks where femtocells are the main base station, the mechanisms to accomplish the load balancing use case could be based on different techniques. Simple and elementary equations [14] can be applied for this use case. However, the best system configuration is usually hard to find. Other mechanisms [17,21] based on FLCs, where efficient rules are defined by experts, present adaptive solutions to reach the best system configuration. Some others propose complex equations or techniques [19], even based on FLCs [18], which lead to high computational cost not really feasible to be implemented in real deployments. In consequence, the main reasons for choosing FLCs are related to the ease for the integration and translation of expert human knowledge into a set of fuzzy rules, its simplicity in implementing and managing rules based on experience, and the low computational cost required to obtain highly improved performance in femtocell networks.

\subsection{Fuzzy logic methodology}

A fuzzy logic controller [22] is a nonparametric approach which provides an efficient and systematic solution for incorporating linguistic information from human experts. The use of rules and interactions make this system relatively easy to understand.

A general fuzzy logic system consists of three stages as Figure 1 illustrates. Firstly, a set of fuzzy membership functions transform crisp inputs into fuzzy input datasets. Secondly, the fuzzy outputs are calculated based on fuzzy input datasets and fuzzy rules (instead of Boolean logic). Finally, those outputs are converted back to crisp values.

The literature offers different implementations of FLCs. This work is focused on the Takagi-Sugeno [23] approach due to its simplicity and computational efficiency. Further details about this approach can be found in the next subsections.

\subsubsection{System parameters and functions}

The system parameters and functions could be basically divided in four groups:

- Linguistic variables: Instead of numerical values (continuous values), the system identifies each input and output variable as one or more linguistic variables (discrete variables), e.g., the base station transmission power adaptation, whose numerical values are ranged between $[-10,10] \mathrm{dB}$, is transformed into a dataset of linguistic terms (sentences or words) such as very negative, negative, zero, positive, and very positive. An

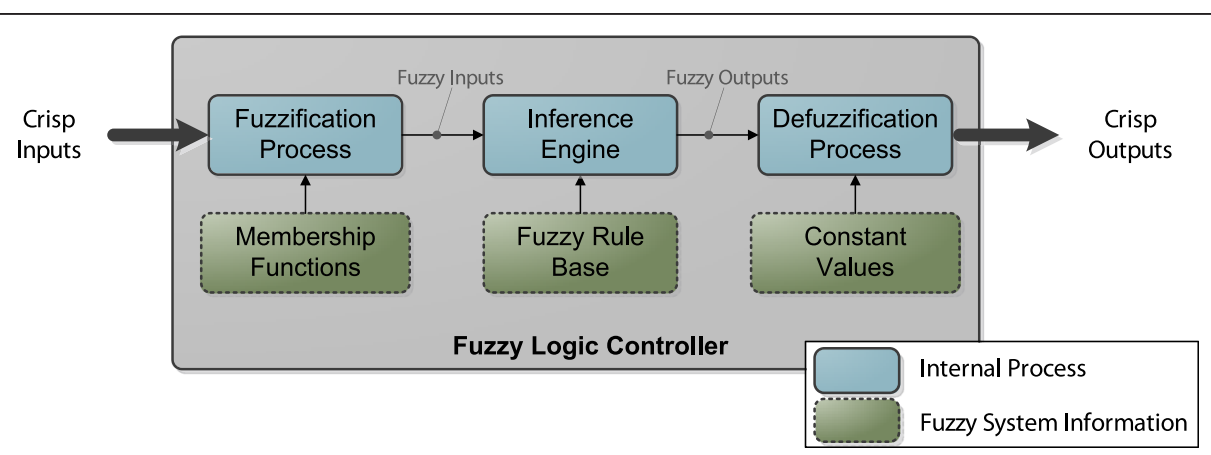

Figure 1 Fuzzy inference system (Takagi-Sugeno [20]). 
example is depicted in Figures 2 and 3 where the available linguistic terms for two different inputs can be found on the top of each function.

- Membership functions: A membership function is a curve that defines the degree of membership of a crisp input (between 0 and 1). Each membership function $\mu_{A}$ $(x)$ is labeled with a linguistic variable. These functions map and quantify crisp inputs $(x)$ from the fuzzy set $A$ into fuzzy input datasets (labeled with linguistic terms). Figures 2 and 3 provide an example of real membership functions. The membership functions to transform fuzzy output datasets into crisp outputs are constant values (for Takagi-Sugeno approach).

- Fuzzy rules: They are simple IF-THEN structures to control the fuzzy output datasets (e.g., transmission power adaptation as very negative, negative, etc.) based on the fuzzy input datasets (e.g., call blocking ratio as very high, high, etc.). A typical fuzzy rule statement, where two crisp inputs are introduced in the system, is similar to the following form:

\section{IF $x$ is $R$ AND $y$ is $S$ THEN $Z$}

where $x$ and $y$ are the crisp inputs, while $R$ and $S$ are the fuzzy input datasets defined in $x$ and $y$, respectively, according to their membership functions. $Z$ is the fuzzy output dataset. Tables 1, 2, and 3 show an example of fuzzy rules.

- Fuzzy set operations: A set of crisp inputs could provide several fuzzy outputs $(Z)$ based on fuzzy rules (e.g., Equation 4). The combination of each rule is carried out using fuzzy set operations, like AND/OR operators in order to get the degree of truth of each rule and finally the crisp output.

\subsubsection{System processes}

The fuzzy logic system is divided into three internal processes as Figure 1 shows. The aim of each module, as well as the flow of information between them, is detailed:

1) Fuzzification process: The fuzzification process is the first step. This process converts crisp inputs into fuzzy input datasets based on the membership functions. These functions quantify the degree of membership of a crisp input $x$ into a fuzzy dataset. For example, at the left bottom of Figure 2, the Load $_{\text {diff }}$ crisp input value $(x=-0.45)$ could be labeled as 'Very Negative' (0.75) and 'Negative' (0.25). These fuzzy input datasets are forwarded to the next step, the inference engine.

2) Inference engine: The fuzzy datasets are evaluated at each rule $r$ to calculate the so-called degree of truth of that rule $\omega_{r}$. Fuzzy set operations accomplish this process. This work follows Equation 4 to generate fuzzy rules, and the product operator (PROD) is selected as the fuzzy set operation for AND intersection operator. The following example proposes two fuzzy input datasets; in consequence, the degree of truth of each rule $r$ is defined as:

$\omega_{r}=\mu_{A}(x) \mu_{B}(y)$

where, $\mu_{A}(x)$ and $\mu_{B}(y)$ are the values of the membership functions for fuzzy sets $A$ and $B$ and crisp inputs $x$ and $y$, respectively.

3) Defuzzification process: After the inference engine, the fuzzy outputs are computed to get the crisp output. For that purpose, there are different defuzzification methods [24].
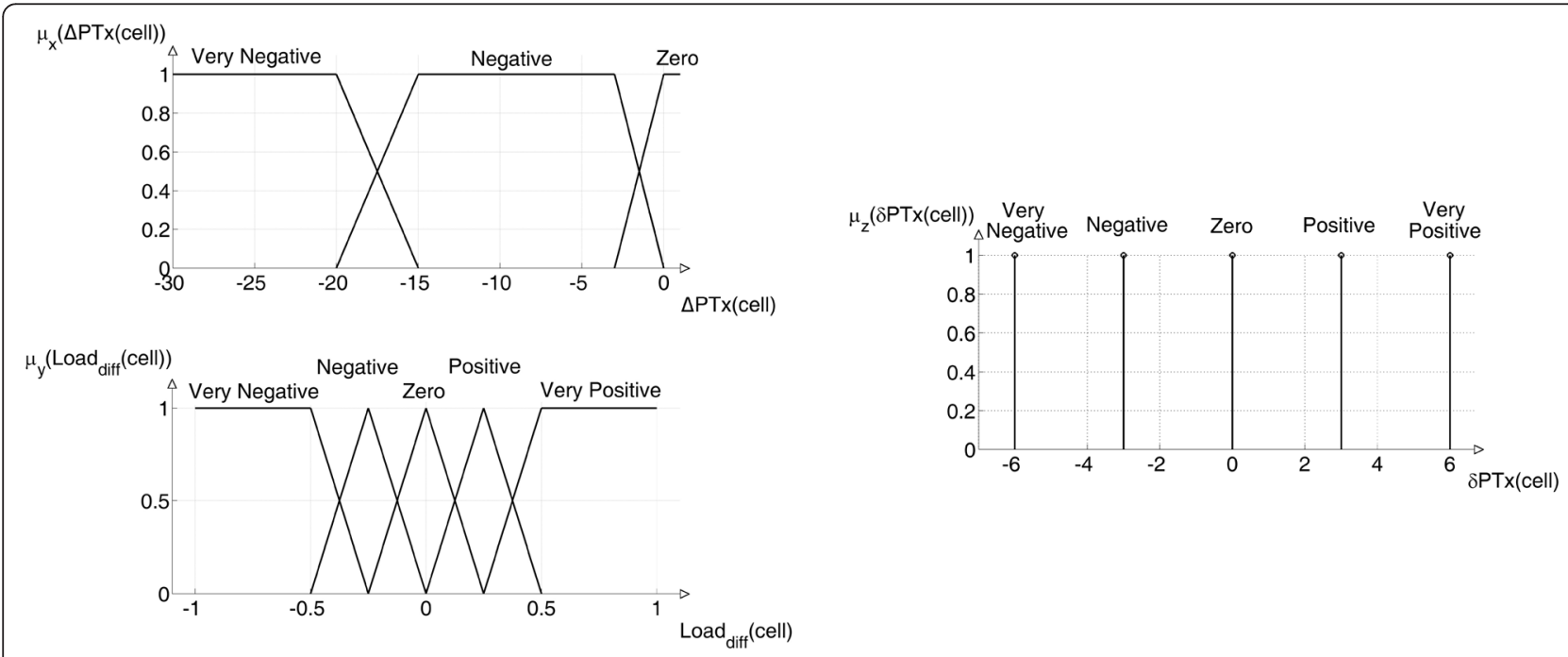

Figure 2 Membership functions of PLS method. 


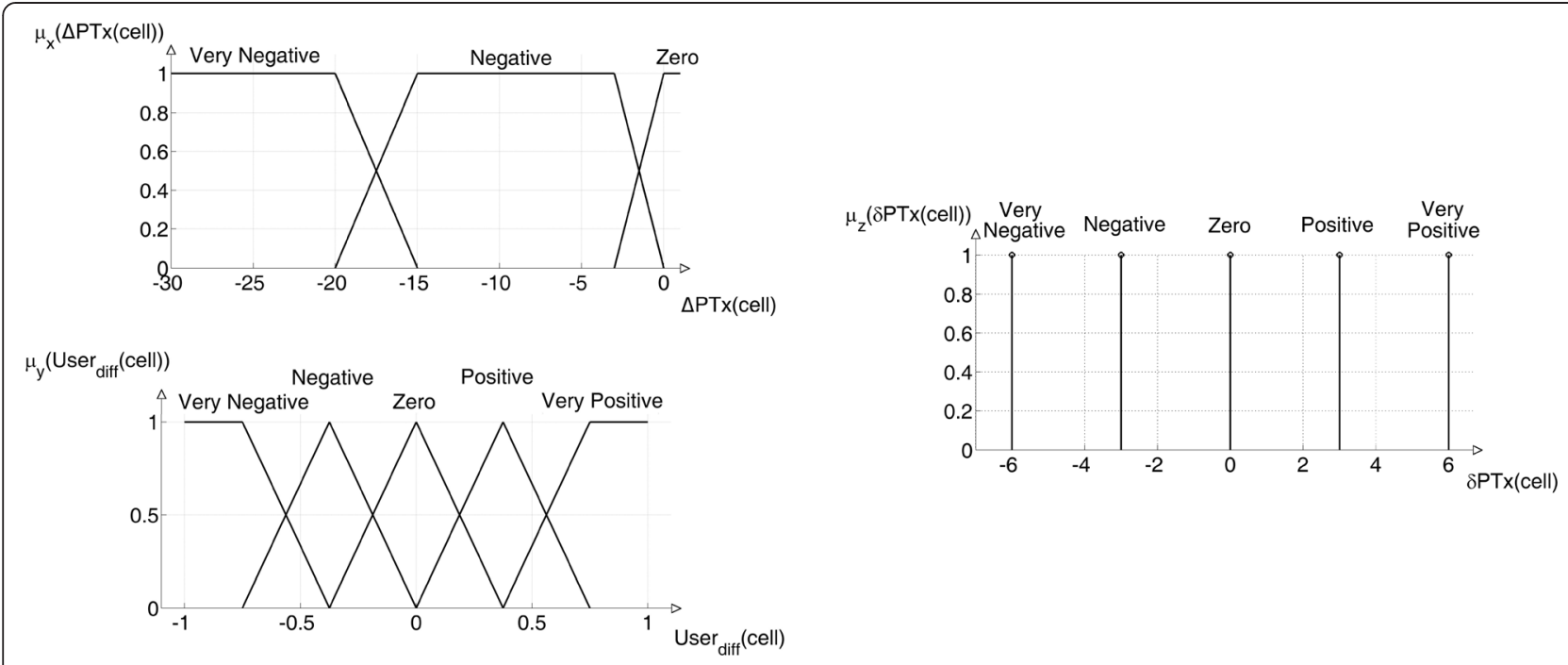

Figure 3 Membership functions of PUS method.

This work is based on the center of gravity for singleton method, that is:

$$
\text { output }=\frac{\sum_{r=1}^{p} \omega_{r} o_{r}}{\sum_{r=1}^{p} \omega_{r}}
$$

where $\omega_{r}$ is the degree of truth of rule $r, o_{r}$ is the constant value of the output variable for the rule $r$, and $P$ is the number of rules.

\section{Load balancing mechanisms}

The basic structure of an FLC applied to optimize cellular communications is depicted in Figure 4. The inputs of these mechanisms can be alarms, counters, and/or key performance indicators (KPIs) acquired from network statistics or call traces, being the collection of this information a standard and usual procedure in cellular

Table 1 Fuzzy rules of PLS method

\begin{tabular}{|c|c|c|c|c|}
\hline & $\begin{array}{l}\text { Load }_{\text {diff }} \\
\text { IF }\end{array}$ & Operator & $\Delta \mathrm{PTx}$ & $\begin{array}{l}\delta P T x \\
\text { THEN }\end{array}$ \\
\hline 1 & Very Negative & AND & Very Negative & Very Positive \\
\hline 2 & Very Negative & AND & Negative & Very Positive \\
\hline 3 & Very Negative & AND & Zero & Positive \\
\hline 4 & Negative & AND & Very Negative & Very Positive \\
\hline 5 & Negative & AND & Negative & Positive \\
\hline 6 & Negative & AND & Zero & Positive \\
\hline 7 & Zero & AND & Very Negative & Very Positive \\
\hline 8 & Zero & AND & Negative & Positive \\
\hline 9 & Zero & AND & Zero & Zero \\
\hline 10 & Positive & AND & - & Negative \\
\hline 11 & Very Positive & AND & - & Very Negative \\
\hline
\end{tabular}

networks. Other inputs, such as a feedback of the outputs, are also of interest. The outputs propose to the cellular network the new reconfiguration parameters.

\subsection{Implementation of controllers}

Following the scheme presented in Figure 4, a reference mechanism as well as a set of additional newly proposed fuzzy-based load balancing methods is detailed below.

The number of input indicators provided to each method is two. Depending on the algorithm, these two input indicators could be classical indicators (such as blocking ratio or cell load), the feedback of the FLC $(\triangle \mathrm{PTx})$, or the newly defined indicator - the ratio of users in connected mode.

In consequence, different membership functions as well as fuzzy rules must be designed according to each input indicator. However, to accomplish consistent and comparable results, all methods utilize the same output information (transmission power deviation per cell, denoted as

Table 2 Fuzzy rules of PUS method

\begin{tabular}{lllll}
\hline & $\begin{array}{l}\text { User } \\
\text { IF }\end{array}$ & Operator & DPTx & $\begin{array}{l}\text { SPTx } \\
\text { THEN }\end{array}$ \\
\hline 1 & Very Negative & AND & Very Negative & Very Positive \\
2 & Very Negative & AND & Negative & Very Positive \\
3 & Very Negative & AND & Zero & Positive \\
4 & Negative & AND & - & Positive \\
5 & Zero & AND & Very Negative & Positive \\
6 & Zero & AND & Negative & Zero \\
7 & Zero & AND & Zero & Zero \\
8 & Positive & AND & - & Negative \\
9 & Very Positive & AND & - & Very Negative \\
\hline
\end{tabular}


Table 3 Fuzzy rules of PLUS method (operator: AND; output: $\delta$ PTx)

\begin{tabular}{llllll}
\hline User $_{\text {diff }}$ & Load $_{\text {diff }}$ & & & & \\
\cline { 2 - 5 } & Very Negative & Negative & Zero & Positive & Very Positive \\
\hline Very Negative & Very Positive & Very Positive & Positive & Zero & Zero \\
Negative & Very Positive & Positive & Positive & Zero & Zero \\
Zero & Very Positive & Positive & Positive & Negative & Very Negative \\
Positive & Zero & Zero & Negative & Negative & Very Negative \\
Very Positive & Negative & Negative & Very Negative & Very Negative & Very Negative \\
\hline
\end{tabular}

$\Delta \mathrm{PTx}($ cell)) and output membership functions (constant values in the studied FLC approach) in the defuzzification process.

\subsubsection{Power traffic sharing - reference}

This method, presented in [17], has been selected as the reference fuzzy-based load balancing algorithm. The FLC inputs are the difference between BR of the analyzed serving cell 'cell' and the average BR of its neighbors (BRdiff (cell)), as well as the femtocell transmission power deviation $\triangle \mathrm{PTx}$ (cell). The latter, $\Delta \mathrm{PTx}$ (cell), is calculated as the maximum femtocell transmission power $\mathrm{PTx}_{\max }$ (cell) minus the current femtocell transmission power PTx (cell) and power adaptation (crisp output $\delta \mathrm{PTx}$ (cell)) as presented in Equation 7. $\delta \mathrm{PTx}$ (cell) presents the transmission power adaptation (increase or decrease) that must be tuned in the femtocell.

$$
\Delta \mathrm{PTx}(\text { cell })=\operatorname{PTx}_{\max }(\text { cell })-[\operatorname{PTx}(\text { cell })+\delta \operatorname{PTx}(\text { cell })]
$$

This optimization procedure improves the performance in heavily loaded enterprise femtocell networks by distributing the users along the unloaded femtocells. Nevertheless, this algorithm is not suitable to solve the issues presented in this paper due to the following reason: the proposed BR indicator provides valuable information once the network already presents an accessibility issue. A prediction of this inconvenient situation would be desirable

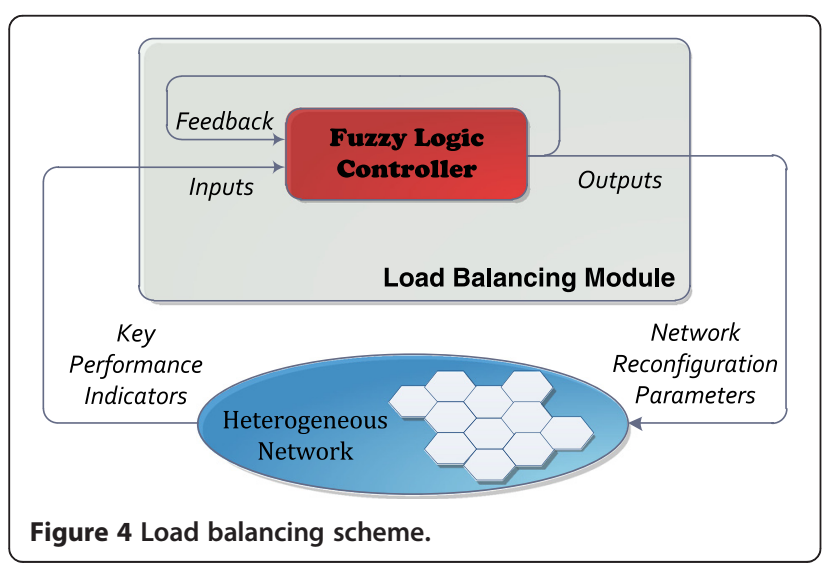

to avoid or reduce users' dissatisfaction as soon as possible. Therefore, this algorithm cannot achieve the optimal network configuration parameter under temporary traffic fluctuations. For further details about the power traffic sharing (PTS) method, the reader is referred to the original work [17].

\subsubsection{Power load sharing}

This algorithm aims to avoid the previous PTS issues. Following the same scheme, the first step is to change the inputs of the algorithm to prevent blocked calls. According to this, the new input indicator is related to bandwidth, by measuring the current cell capacity in radio resource terms. This indicator is hereafter described for LTE networks, although equivalent indicators can be defined for other technologies. Therefore, in power load sharing (PLS), the load balance is performed based on the occupied physical resource block (PRB), whereas the previous PTS optimizer depended on blocked calls. The PRB is a basic unit of LTE radio resources that provides instantaneous information about cell load. According to this, the key network indicator for this system is represented as the following function, which is calculated for each cell and FLC:

$$
\operatorname{Load}_{\text {diff }}(\operatorname{cell})=\operatorname{Load}(\operatorname{cell})-\frac{1}{N} \sum_{i=1}^{N} \operatorname{Load}(i)
$$

where Load (cell) and Load (cell) are the ratio of occupied PRBs of the studied cell (serving cell) and the neighboring cells, respectively, and $N$ is the number of neighboring cells. This indicator is used as FLC input together with the femtocell transmission power deviation $\triangle \mathrm{PTx}$ (cell) (see Equation 7). A similar method was originally presented in [16], although the PLS proposed hereafter modifies the membership functions and fuzzy rules compared to [16] to be suitable for solving temporary overloaded situations. In this context, based on the expert knowledge, some membership functions and fuzzy rule configurations have been evaluated. A large enough number of membership functions has been selected to achieve a reasonable level of detail while keeping the number of fuzzy sets small enough to build easy sets of fuzzy rules. The proposed membership functions 
are shown in Figure 2. On the left top image, three membership functions $\mu_{x}(\Delta \mathrm{PTx}($ cell $))$ are designed to analyze if current power deviation is very negative 'Very Negative, negative 'Negative,' or zero 'Zero.' The function $\mu_{y}\left(\operatorname{Load}_{\mathrm{diff}}(\mathrm{cell})\right)$, on the left bottom of Figure 2, depicts five membership functions to characterize the inputs in 'Very Negative,' 'Negative,' 'Zero,' 'Positive', and 'Very Positive' according to the difference between the studied cell load ratio and the ratio of its neighboring cells, keeping the symmetry around 0 (i.e., the point where the load between the studied cell and its neighbors is the same) to balance the load in the network. Each membership function is defined in an interval based on the expert knowledge, and for simplicity and computational efficiency, the selected membership functions are trapezoidal and triangular.

Table 1 defines the common sense fuzzy rules in the form of IF-THEN statements, following the syntax of Equation 4. These rules are built to balance the ratio of the studied cell load and its neighboring cell load. In consequence, the more positive the $\operatorname{Load}_{\text {diff }}$ (cell) is (i.e., the studied cell is overloaded), the more negative the transmission power deviation should be (rules 10 and 11 ), and vice versa (rules 1 to 6 ). In case the network is balanced $\left(\operatorname{Load}_{\text {diff }}=\right.$ 'Zero'), the algorithm tends to return to the initial transmission power configuration in order to improve SINR and reduce the number of PRBs per user as the channel quality indicator (CQI) is increased (rules 7 to 9). In consequence, the cell would have more available free resources decreasing the probability of cell congestion.

Based on the output function $\mu_{z}(\delta \mathrm{PTx}$ (cell) shown on the right of Figure 2, the femtocell transmission power is increased or decreased $\delta \mathrm{PTx}$ (cell). The functions can take the following labels and values: 'Very Negative' and $-6 \mathrm{~dB}$, 'Negative' and $-3 \mathrm{~dB}$, 'Zero' and $0 \mathrm{~dB}$, 'Positive' and $+3 \mathrm{~dB}$, and 'Very Positive' and $+6 \mathrm{~dB}$. Finally, the femtocell transmission power $\mathrm{PTx}($ cell $)$ is tuned according to the calculated $\delta \mathrm{PTx}(\mathrm{cell})$.

This method has been defined focusing on an LTE network, although it could be easily applied to any other cellular technology. The PRB is bandwidth-related; therefore, an equivalent available bandwidth indicator can be used as input for any other cellular technology.

\subsubsection{Power user sharing}

Femtocells are usually designed to support $4,8,16$, or 32 users in connected mode, either voice or data traffic [25]. Rather than a wireless cellular bandwidth limitation, this characteristic is a restriction in the femtocell processing capability. For that reason, PRB activity (as proposed in the PLS method) may not be always an appropriate indicator for balancing traffic in femtocell environments where most of the time there could be free resources to allocate user data, but the femtocell is not able to process them due to the limit in the number of accepted users. According to this, the proposed power user sharing (PUS) method considers the number of users in connected mode as the main parameter in order to offload temporary congested cells.

In this case, the input indicator of the FLCs is defined as the ratio of users in connected mode, i.e., the number of simultaneous users in connected mode $N_{\text {active _users }}$ to the femtocell user limitation $N_{\text {femto _ user_limitation }}$ (see Equation 10) of the studied cell User (cell), in relation to the same ratio averaged in its neighboring cells User $(i)$ :

$$
\operatorname{User}_{\text {diff }}(\text { cell })=\operatorname{User}(\text { cell })-\frac{1}{N} \sum_{i=1}^{N} \operatorname{User}(i)
$$

where $N$ is the number of neighboring cells and User $(c)$ is defined for a cell $c$ as:

$$
\operatorname{User}(c)=\frac{N_{\text {active_users }}(c)}{N_{\text {femto_user_limitation }}(c)}
$$

This mechanism presents the same FLC structure as the previous ones, but the inputs of the FLC are the new indicator, User $_{\text {diff }}$ (cell) as presented in Equation 9, and the femtocell transmission power deviation, $\Delta \mathrm{PTx}$ (cell). The output of the FLC, $\delta \mathrm{PTx}$ (cell), increases/decreases the current femtocell transmission power.

In order to get a moderate level of detail and straightforward fuzzy control rules, an acceptable number of membership functions have been selected for each indicator based on experienced human knowledge. The membership functions of this controller are shown in Figure 3. As it can be observed, the membership functions $\mu_{x}(\Delta \mathrm{PTx}($ cell $))$ are the same as the previous membership functions of the PLS method (left top image of Figure 3), but the new input User $_{\text {diff }}($ cell) associates different membership functions $\mu_{y}\left(\mathrm{User}_{\text {diff }}(\right.$ cell $\left.)\right)$ according to the difference between the ratio of users in the studied cell and the ratio of its neighboring cells at the left bottom of Figure 3: 'Very Negative,' 'Negative, 'Zero,' 'Positive', and 'Very Positive'. Notice that these membership functions are similar to the previous method; however, the interval of definition is adjusted by experts to get the best algorithm's performance. For simplicity and computational efficiency, the implemented membership functions are triangular and trapezoidal.

Fuzzy rules set the control strategy based on Equation 4 . These rules are depicted in Table 2 and aim to reach equilibrium in the ratio of active users in the studied cell and the ratio of its neighboring cells. According to this, the more positive $U_{s e r}$ diff $(c e l l)$ is (i.e., the ratio in the studied cell is higher), the more negative the transmission power deviation should be (rules 8 and 9), and vice versa (rules 1 to 4). Once the network is equalized $\left(\mathrm{User}_{\text {diff }}(\right.$ cell $)=$ 'Zero' $)$, 
the PUS method avoids very negative transmission power adaptations (label 'Very Negative' - rule 5) in order to increase the end-users' QoS. However, as this method is restricted by the number of users (instead of the available resources in the cell), returning to the default transmission power could increase the interference, but the cell congestion would not be reduced. That is why 'Negative' or 'Zero' values of transmission power deviation (rules 6 and 7) do not return to the default value.

The same output functions $\mu_{z}(\delta \mathrm{PTx}($ cell)), as in the previous algorithm, are defined for this one (illustrated on the right of Figure 3). The labels and values are as follows: 'Very Negative' and $-6 \mathrm{~dB}$, 'Negative' and $-3 \mathrm{~dB}$, 'Zero' and $0 \mathrm{~dB}$, 'Positive' and $+3 \mathrm{~dB}$, and 'Very Positive' and $+6 \mathrm{~dB}$.

Most of the time, this mechanism is suitable for achieving good performance in femtocell networks, above all in LTE deployments where the bandwidth is, nowadays, high enough to ensure the end-users' QoS. However, it could present some shortcomings when the propagation channel is very poor (bad SINR) or users need to download the best effort data.

\subsubsection{Power load and user sharing}

The previous mechanisms, as explained, could not guarantee their proper operation in some situations. The shortcomings of each algorithm (PLS and PUS methods) could be complemented by the other, leaving outside the PTS method due to the long time needed to evaluate its main indicator (BR). That means, a combination of the two indicators (cell load and number of active users) is mandatory to ensure an efficient mobility load balancing procedure in indoor heterogeneous femtocell networks. According to this, the works described in the literature in the context of MLB at femtocell networks that did not analyze at least these two indicators do not properly work as it will be demonstrated in Section 5.

This paper proposes an FLC algorithm whose inputs are both previously defined KPIs User ${ }_{\text {diff }}($ cell) and Load diff (cell) to properly prevent or reduce occasional indoor congestions. For simplicity, this method does not include the FLC feedback as an input indicator.

The same membership functions proposed for PLS and PUS methods are applied to this FLC in order to verify the benefits of their combination and to proceed with their comparison under the same conditions (readers are referred to the left bottom images of Figures 2 and 3 as reminder of those functions).

New fuzzy rules are defined based on these two indicators and the expert knowledge. Table 3 presents the set of control rules implemented in this system. These rules

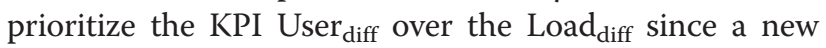
user in a femtocell network usually implies a greater impact on the maximum allowed number of user in a femtocell than on the available resources. That means the network resources assigned to each user could be reduced in order to accept new users, even if some of them could be slightly dissatisfied (decrease of QoS, throughput, etc.). For example, under a 'Very Negative' situation of Load $_{\text {diff }}$ and a 'Very Positive' situation of

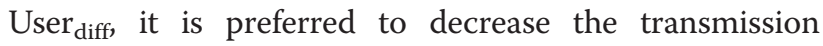
power of the studied cell (fuzzy output is 'Negative').

The same constant output functions $\mu_{z}(\delta \mathrm{PTx}(\mathrm{cell}))$, as in the previous algorithms, are applied here. In order to compare all the algorithms under the same conditions, the labels and values are as follows: 'Very Negative' and $-6 \mathrm{~dB}$, 'Negative' and $-3 \mathrm{~dB}$, 'Zero' and $0 \mathrm{~dB}$, 'Positive' and $+3 \mathrm{~dB}$, and 'Very Positive' and $+6 \mathrm{~dB}$.

A summary of the supported inputs and outputs of the proposed load balancing mechanisms is depicted in Table 4.

\section{Performance analysis}

The evaluation of the presented mechanisms is explained in this section. On the one hand, an introduction to the simulation tool and the selected scenario is provided. On the other hand, the sensitivity of the algorithm's performance with regard to the changes in the membership functions is covered, and the assessment of the algorithm performance is evaluated.

\subsection{Analysis setup}

The load balancing mechanisms are evaluated in a dynamic LTE system-level simulator described in [26]. Winner II [27] was the implemented propagation model at the simulator, as several propagation configurations are considered for indoor, outdoor, outdoor-to-indoor, and indoor-to-outdoor scenarios. Likewise, shadowing and fast fading are modeled following a spatially correlated log-normal distribution with different standard deviations for outdoor and indoor users and the extended indoor A (EIA) approach for indoor users [28], respectively. Users can demand both VoIP or data services in the scenario, where half of the PRBs are reserved for VoIP and the other half for data service. In case the number of PRBs is odd, the last PRB is assigned to data service. Round-robin best channel scheduling is selected for VoIP traffic and proportional fair scheduling for data traffic. Common radio resource management (RRM) features are also integrated into the simulator, such as cell

Table 4 Summary of load balancing mechanisms

\begin{tabular}{lllll}
\hline & PTS $^{\text {a }}$ & PLS & PUS & PLUS \\
\hline Input 1 & $\Delta P T x$ & $\Delta P T x$ & $\Delta P T x$ & Load diff \\
Input 2 & BR $_{\text {diff }}$ & Load $_{\text {diff }}$ & User diff & User diff $^{\text {d }}$ \\
Output & $\delta P T x$ & $\delta P T x$ & $\delta P T x$ & $\delta P T x$ \\
\hline
\end{tabular}

Reference [14]. 
reselection $(\mathrm{CR})$, directed retry $(\mathrm{DR})$, and $\mathrm{HO}$ based on 'A3' and 'A5' events. The most important simulation parameters and features are shown in Table 5. A full simulator description can be found at reference [26].

A real airport departure area (Malaga Airport) has been selected and designed as a realistic simulation scenario to assess the proposed mechanisms. It is a 265 -m-long and 180-m-wide building, which is placed into a 3-km-long and 2.6-km-wide area, as Figure 5 shows. A tri-sectorized LTE macrocell is located in that scenario, and twelve open access femtocells (HeNB) are distributed along the building to ensure the end-users' QoS and good coverage at each location by at least two femtocells. Outdoor macrocells introduce indoor interference due to the building proximity to the sites. Handovers between macrocells and femtocells are also supported. At the airport, the user mobility is based on a random waypoint model [29], which reproduces normal passenger behavior and movement at the airport. Passengers are located to create a hotspot and to overload a specific femtocell during certain minutes, emulating a delayed boarding (passengers use their

Table 5 Simulation parameters

\begin{tabular}{|c|c|c|}
\hline \multirow{2}{*}{$\begin{array}{l}\text { Parameter } \\
\text { Propagation model }\end{array}$} & \multicolumn{2}{|l|}{ Configuration } \\
\hline & Indoor-indoor & Winner II A1 \\
\hline & Indoor-outdoor & Winner II A2 \\
\hline & Outdoor-outdoor & Winner II C2 \\
\hline & Outdoor-indoor & Winner II C4 \\
\hline \multirow[t]{3}{*}{ Base station model } & EIRP & 3 (HeNB)/43 (macro) dBm \\
\hline & Directivity & Omni (HeNB)/tri-sector (macro) \\
\hline & Access & Open (HeNB)/open (macro) \\
\hline \multirow{2}{*}{$\begin{array}{l}\text { Mobile station } \\
\text { model }\end{array}$} & Noise figure & $9 \mathrm{~dB}$ \\
\hline & Noise density & $-174 \mathrm{dBm} / \mathrm{Hz}$ \\
\hline \multirow[t]{2}{*}{ Traffic model } & Calls & Poisson (avg. 0.43 calls/user $\cdot h$ ) \\
\hline & Duration & Exponential (avg. 100 s) \\
\hline \multirow[t]{2}{*}{ Mobility model } & Outdoor & $\begin{array}{l}3 \mathrm{~km} / \mathrm{h} \text {, random direction and } \\
\text { wrap-around }\end{array}$ \\
\hline & Indoor & Random waypoint \\
\hline \multirow[t]{2}{*}{ Service model } & Voice over IP & $16 \mathrm{kbps}$ \\
\hline & Full buffer & \\
\hline \multirow[t]{6}{*}{ RRM model } & Bandwidth & $\begin{array}{l}\text { 1.4, 3, and } 5 \mathrm{MHz}(6,15 \text {, and } \\
25 \text { PRBs) }\end{array}$ \\
\hline & Access control & Directed retry (threshold $=-44 \mathrm{dBm}$ ) \\
\hline & Cell reselection & Criteria S, R \\
\hline & Handover & Events $\mathrm{A} 3, \mathrm{~A} 5$ \\
\hline & Scheduler & Voice: round-robin best channel \\
\hline & & Full buffer: proportional fair \\
\hline Time resolution & & $100 \mathrm{~ms}$ \\
\hline $\begin{array}{l}\text { Load balancing } \\
\text { algorithm }\end{array}$ & Epoch time & $60 \mathrm{~s}$ \\
\hline
\end{tabular}

smartphones waiting for boarding). That situation could cause temporary network congestions, therefore, occasional accessibility or retainability issues.

\subsection{Simulation results}

Firstly, a sensitivity study is performed to analyze the performance of the algorithms with regard to the changes in the membership functions. Secondly, the global network and hotspot (most congested cell) simulation results are presented and described for each method in a specific scenario: femtocells limited to eight simultaneous connected users and 1.4-MHz bandwidth. Thirdly, the study is extended to different femtocells' capacity deployments $(4,8$, 16 , and 32 users each) and bandwidth (1.4, 3, and $5 \mathrm{MHz}$ ).

The simulated scenarios are initially well-balanced and there are no congestion issues. After several minutes, new passengers arrive to the boarding gate, thus overloading the femtocell in charge of covering that area. That situation lasts several minutes until the passengers of the delayed flight can start boarding and network congestion issues disappear. To evaluate the whole process, the simulation is carried out along $1 \mathrm{~h}$, whereas the selfoptimization algorithms analyze the network every $60 \mathrm{~s}$ to decide whether they must be triggered or not.

The evaluation of these scenarios is analyzed from both the user's QoS perspective (BR and OR indicators) and operator point of view (HOR indicator). To simplify the assessment of end-user happiness with the mobile service, the user dissatisfaction ratio (UDR) [17] has been selected, which measures the percentage of dissatisfied users as a linear combination of BR and OR:

$$
\mathrm{UDR}=\mathrm{BR}+(1-\mathrm{BR}) \cdot \mathrm{OR}
$$

\subsubsection{Sensitivity of the algorithm's performance}

Diverse sets of membership functions were proposed by experts for each input indicator ( $\operatorname{Load}_{\text {diff }}$ and $U_{s e r}{ }_{\text {diff }}$ ). Additionally, other membership functions out of these recommendations were evaluated. In this sense, a sensitivity study is performed in the previous scenario where the maximum number of simultaneous connections is restricted to four users and the femtocell bandwidth is 1.4 MHz. Table 6 shows the performed sensitivity study where the UDR indicator has been evaluated. On the one hand, the analysis shows that in any case the algorithm's performance was not very sensitive to the set of membership functions proposed by experts (see the evaluation of set 1 to set 5 in Table 6). On the other hand, membership functions different to the recommendations of experts (set 6 to set 10 in Table 6) show (as expected) lower system performance. Therefore, it is assumed that membership functions that significantly 


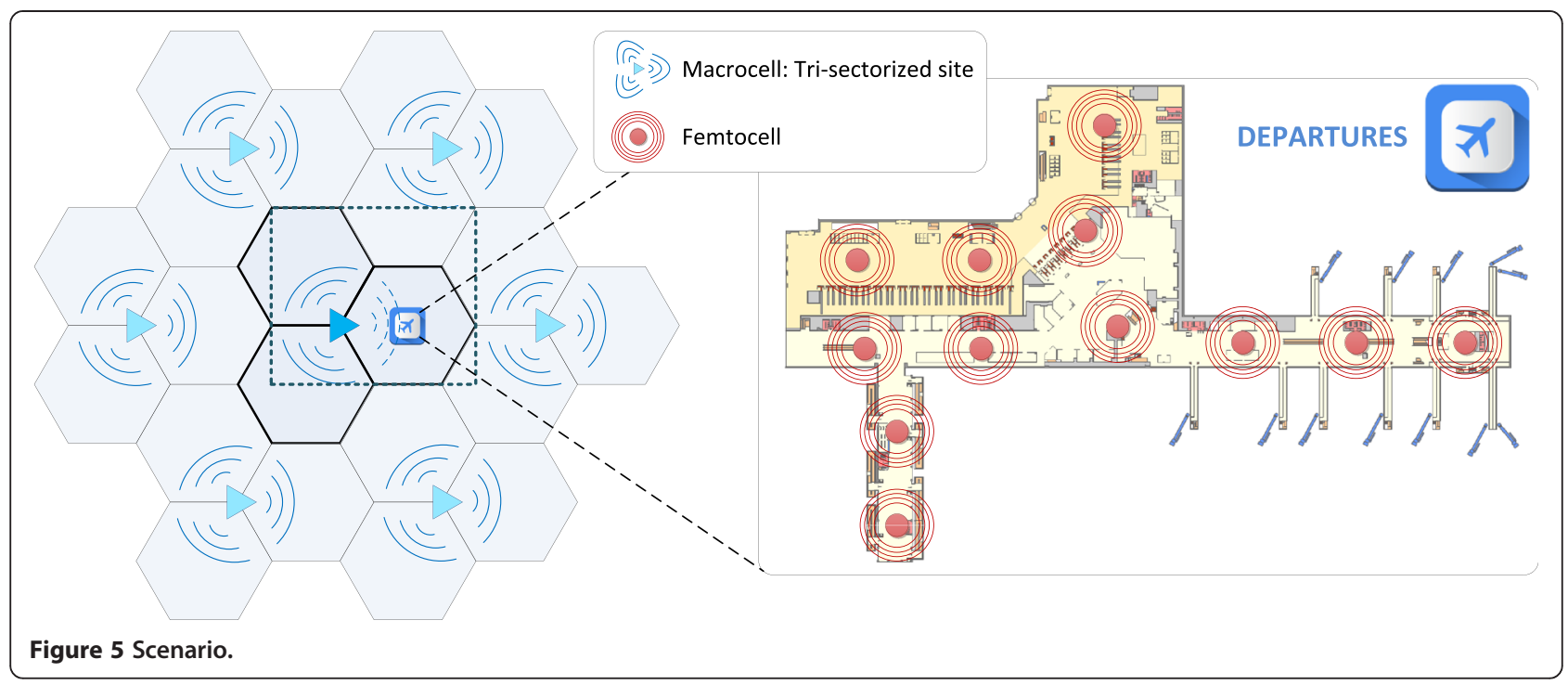

differ from experts' recommendations would probably decrease the algorithm's performance.

Finally, the set of membership functions with the best solution is implemented (functions presented in Figures 2 and 3). These membership functions provide the best results of the evaluated cases for Loaddiff and Userdiff inputs. Therefore, they are the ones in which evaluation is described in detail in the next subsections.

\subsubsection{Scenario: maximum eight users/femtocell at $1.4 \mathrm{MHz}$}

The first analysis corresponds to the situation in which all femtocells limit is set to eight users and the network bandwidth is $1.4 \mathrm{MHz}$, i.e., it supports six PRBs. Both, the global network and the hotspot performance are assessed in these tests.

Figure 6a illustrates the UDR indicator during 1-h simulation. This indicator is collected every minute as vertical lines depict. This figure represents the network analysis when there is no optimization method implemented at this scenario (no OPT - blue stem) and the performance of the four load balancing algorithms. The average value during the simulation is also shown for each method.

The non-optimized situation (blue stem), which represents the absence of load balancing methods, shows no congestion issues at the beginning and the end of the evaluation, i.e., no users are dissatisfied. However, the network displays a high percentage of dissatisfied users during the overloaded period ( 30 to $50 \mathrm{~min}$ ). Sometimes, the ratio of dissatisfied users reach UDR $=50 \%$, i.e., half of the users that attempt to access the network are rejected or connected users are in outage. On average, this network indicator is $10 \%$, which is higher than the values typically accepted by mobile operators (horizontal blue line).

The PTS method (red stem) starts to reduce the blocked calls after detecting them; therefore, the UDR decreases as well. Nevertheless, due to the traffic fluctuations, peaks of UDR are obtained. Thanks to this mechanism, the average UDR is reduced to about $4 \%$. However, as previously explained, this mechanism needs a large period of time to converge under the same network conditions. Hence, although the indicator is improved, BR is not the proper input to optimize these situations.

The PLS mechanism (green stem) provides improved results because it makes use of the occupied radio resources as input, decreasing the number and the value of dissatisfied users' situations. The horizontal green line in Figure $6 \mathrm{a}$ shows an improvement over $7 \%$ from the non-optimized situation and 1\% from the PTS method, getting an average value of UDR below $3 \%$. Nevertheless, its Load diff $_{\text {(cell) in- }}$ put does not reflect the most restricted factor for femtocells because the system bandwidth usually offers enough

Table 6 Sensitivity analysis (UDR [\%])

\begin{tabular}{|c|c|c|c|c|c|c|c|c|c|c|}
\hline & \multicolumn{10}{|c|}{ Max. four users/femtocell (1.4 MHz) } \\
\hline & Set 1 & Set 2 & Set 3 & Set 4 & Set 5 & Set $6^{\mathrm{a}}$ & Set $7^{a}$ & Set $8^{a}$ & Set $9^{a}$ & Set $10^{\mathrm{a}}$ \\
\hline PLS & 3.6458 & 3.6594 & 3.6487 & 3.6624 & 3.6548 & 5.157 & 5.2368 & 5.4861 & 5.1958 & 5.3682 \\
\hline PUS & 1.2351 & 1.241 & 1.2115 & 1.2254 & 1.2388 & 4.1253 & 4.3672 & 4.6985 & 4.1687 & 4.8372 \\
\hline
\end{tabular}

${ }^{\mathrm{a}} \mathrm{Set}$ of membership functions not defined by experts. 


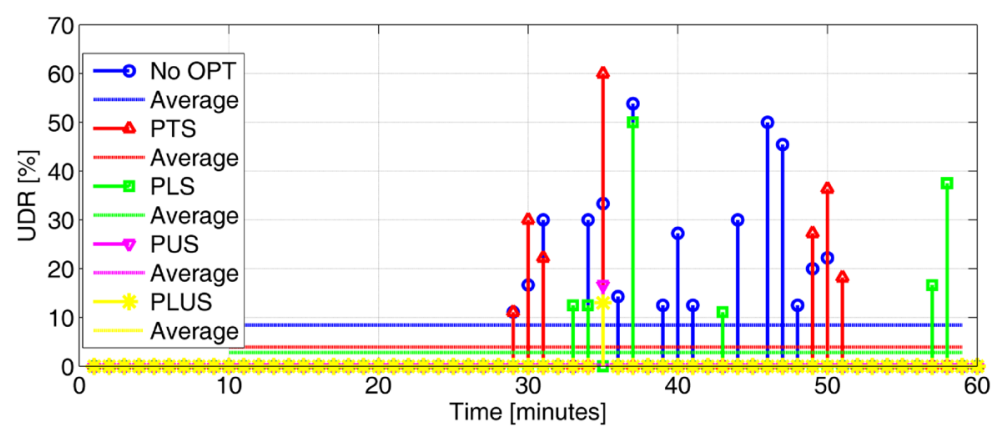

a)

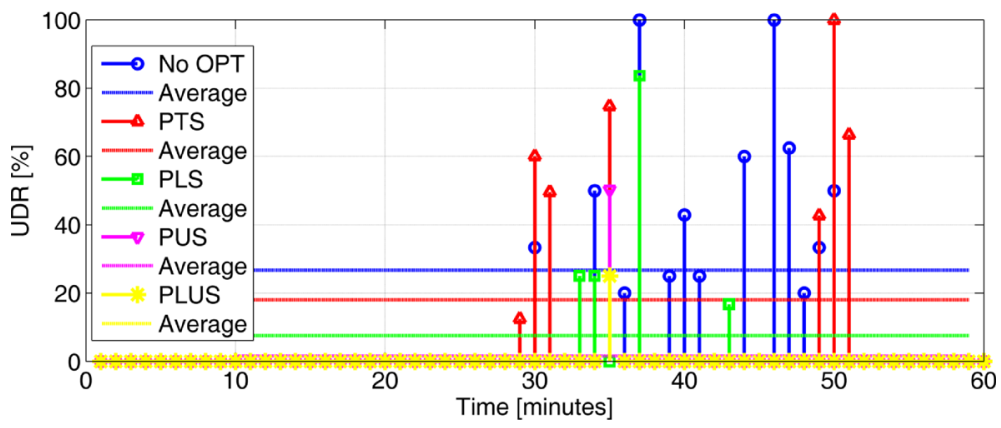

b)

Figure 6 UDR performance. (a) Global network performance (eight users/femtocell and 1.4 MHz). (b) Hotspot performance (eight users/femtocell and $1.4 \mathrm{MHz}$ ).

capacity to serve more than the femtocell capability in terms of the maximum number of users in connected mode, i.e., the self-optimization algorithm is not triggered (free radio resources) but the femtocell is totally congested (no more users are accepted). Therefore, the PLS method does not reach the best possible results for the optimization process.

The PUS method (magenta stem) reduces even further the users' dissatisfaction. However, the performance of the algorithm decreases when the required user's QoS is very high (huge amount of radio resources are demanded by the user). In this case, the small amount of users (max. eight users) and the wide bandwidth, at this example, reduce the users' dissatisfaction. With this method there are only problems for an instant (35 $\mathrm{min}$ ), where few users are connected to the network but a lot of radio resources are required. A very low average UDR is obtained, lower than $1 \%$ (horizontal magenta line).

The power load and user sharing (PLUS) method (yellow stem), as expected, provides the most improved results. In this case, the algorithm is aware of the exact amount of free space, both in terms of femtocell hardware capacity and radio resources, to access the femtocell. Therefore, it is able to modify network parameters when any of them (according to fuzzy rules) presents high values. The figure shows the same problem as with
PUS method for an instant (35 min), where few users are connected to the network but a lot of radio resources are required. However, the UDR is lower. In average during $1 \mathrm{~h}$ (horizontal yellow line), the value of UDR is around $0.2 \%$, which complies with the usual operators' requirements.

UDR indicator at the most overloaded cell, i.e., at the hotspot, is shown in Figure 6b. UDR evolves in the same way as in Figure 6a, although it is higher in all cases. In average, around $30 \%$ of users are dissatisfied, while the optimization PTS, PLS, PUS, and PLUS methods get around $18 \%, 7 \%, 2 \%$, and $1 \%$ of UDR, respectively.

\subsubsection{Average network performance: remaining scenarios}

The previous study has been extended to other femtocell capability deployments (i.e., maximum number of connected users) and different bandwidth. The performance in terms of the average value of UDR is depicted in Figure 7 for the different bandwidth and user limitations considered.

As worst case, the minimum LTE bandwidth $(1.4 \mathrm{MHz})$ has been selected in the first subfigure of Figure 7a. It can be observed that the UDR is reduced by the implementation of the load balancing methods for all 4, 8, 16, and 32 users/femtocell users' limitation. For the scenario of 32 users/femtocell, depending on the experienced users' 


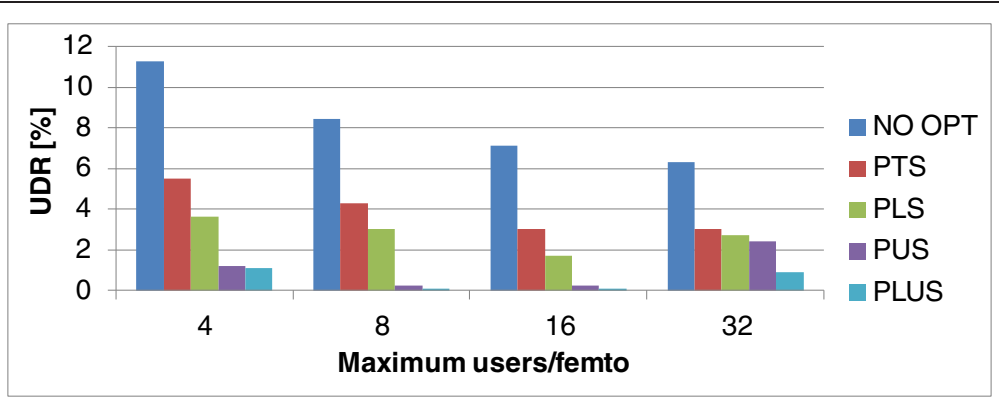

a)

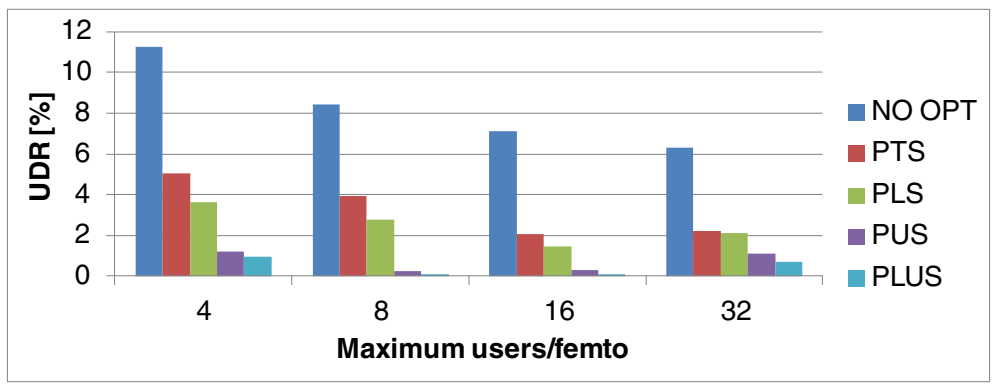

b)

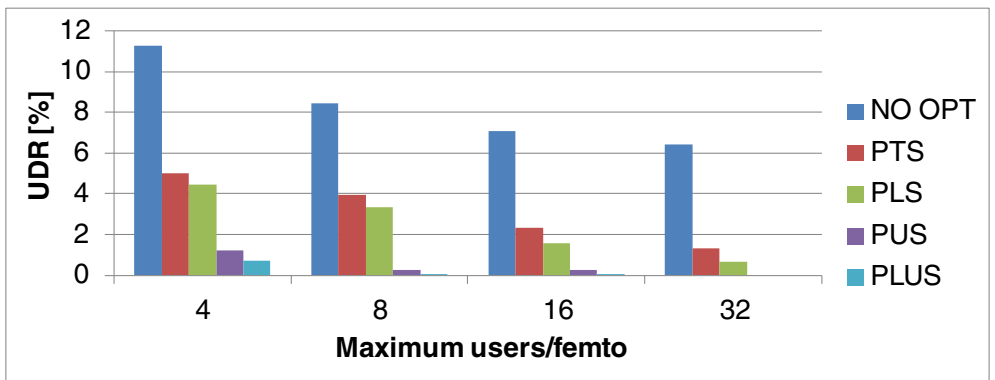

c)

Figure 7 Average UDR performance. (a) $1.4 \mathrm{MHz}$ (6 PRBs). (b) $3 \mathrm{MHz}$ (15 PRBs). (c) $5 \mathrm{MHz}$ (25 PRBs).

quality and demand, sometimes the femtocell allows less than 32 simultaneously connected users because there are no available resources. Likewise, some other times the femtocell allows 32 users even if they are not using all radio resources. The reason is related to the RRM configuration. According to this, all the algorithms have comparable average UDR value except the PLUS method. Those situations overload femtocells adjacent to the hotspot, and as a result, many handover attempts fail (dropped calls and outage connections appear) because those adjacent cells are already fully occupied (users or radio resources). It means that the PUS method will not properly work all the time, the same for the PLS method. However, this issue is successfully fixed, thanks to the PLUS method that combines both network indicators. Additionally, commercial femtocells could avoid this problem because most of them work with wider bandwidth (normally $>3 \mathrm{MHz}$ ).

In this sense, similar simulations have been carried out with higher bandwidth $(3 \mathrm{MHz})$, as Figure $7 \mathrm{~b}$ depicts.
This time, the 32 users/femtocell deployment has enhanced UDR, and in particular, the PUS method presents much better performance compared to the $1.4 \mathrm{MHz}$ case because the network available radio resources are higher. For the other femtocell user limits, the network performance is similar to the previous bandwidth $(1.4 \mathrm{MHz})$ deployments.

In the following test, the network bandwidth was increased to $5 \mathrm{MHz}$. Figure 7c shows the overall network improvement achieved, thanks to the PLUS method.

It should be noticed that the higher the network bandwidth, the worse the PLS method works. This is due to the fact that the network bandwidth is increased but the occupied radio resources are the same (for most network services).

An indicator which concerns operators is the $\mathrm{HO}$ signaling costs (measured by the HOR). The higher the number of handovers the network manages, the more expenses the operator should afford. In Figure 8, the 


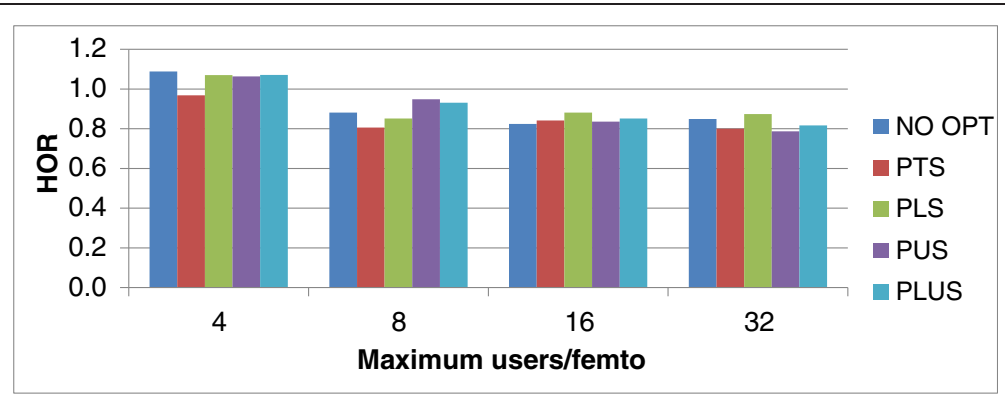

a)

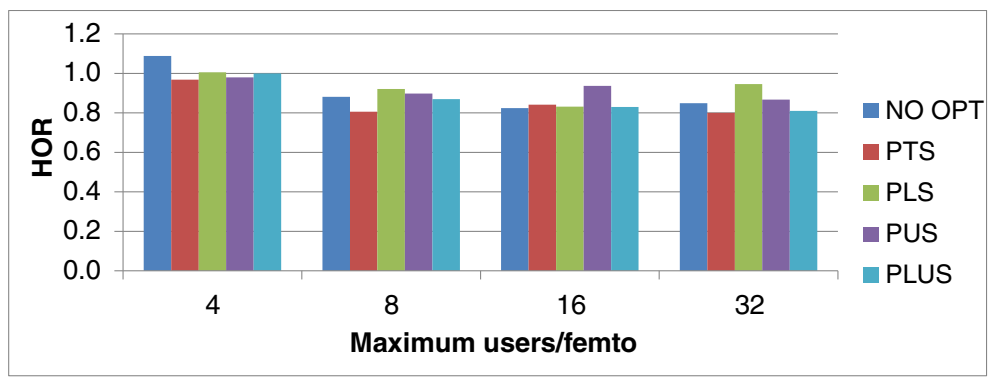

b)

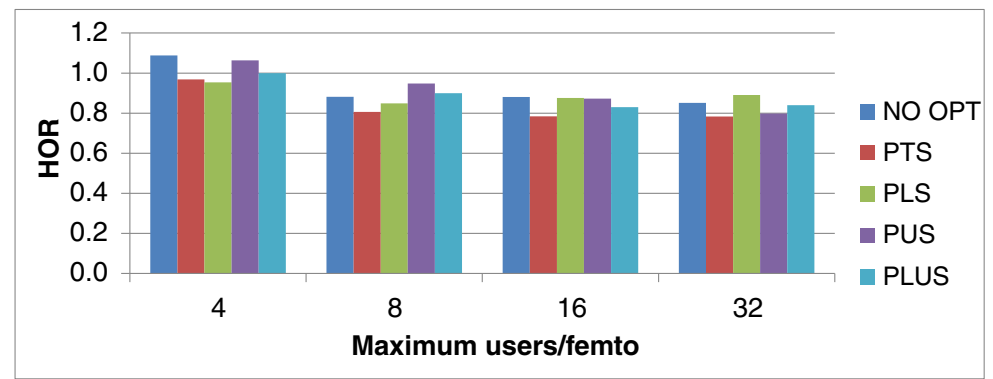

c)

Figure 8 Average HOR performance. (a) $1.4 \mathrm{MHz}$ (6 PRBs). (b) $3 \mathrm{MHz}$ (15 PRBs). (c) $5 \mathrm{MHz}$ (25 PRBs).

results have shown that, in average, there is around one handover per user for all the methods. Hence, changing and resizing femtocell areas do not increase signaling load; in consequence, operator expenses are the same.

To summarize, all methods have reduced end-users' dissatisfaction while the network signaling load is held. In conclusion, at indoor heterogeneous networks where different bandwidth and types of femtocells are analyzed, both femtocell radio resources and femtocell users' restriction related to the femtocell hardware must be considered in mobility load balancing methods. The proposed PLS and PUS methods provide good results, but their actual performance depends on the predominant kind of traffic. The proposed PLUS method however led to the best network and hotspot performance for all situations with fast traffic fluctuations.

\section{Conclusions}

In this paper, novel and low-complexity load balancing methods based on fuzzy logic controllers have been proposed and analyzed for temporary congested indoor heterogeneous femtocell scenarios.

It has been shown that load balancing algorithms with classical input indicators improved the network performance (compared to the non-optimized situation) while increasing the coverage and capacity in indoor heterogeneous femtocell environments. However, in previous references, these mechanisms were evaluated under very specific simulated situations, as proven by the limitations of the PTS or PLS methods presented in the results. Conversely, this work has proposed a simple but powerful parameter, the number of simultaneous connected users per femtocell, as an indicator that must be taken into account in these scenarios. However, the 
proposed PUS method based uniquely on such indicator and transmission power deviation shows benefits only under specific conditions (i.e., the number of users is over femtocell limit), whereas in other cases (i.e., the low number of users over femtocell limit but high femtocell load), it presents some shortcomings that degrade the network performance. Finally, the PLUS method that integrates classical indicators and this new one has been proved as the best combination to build a consistent and reliable load balancing mechanisms in indoor heterogeneous femtocell environments.

\section{Abbreviations}

3GPP: Third Generation Partnership Project; BR: blocking ratio; CAPEX: capital expenditure; CQI: channel quality indicator; CR: cell reselection; DR: directed retry; EIRP: equivalent isotropically radiated power; FLC: fuzzy logic controllers; HCN: heterogeneous cellular networks; HeNB: home eNB; HO: handover; HOR: handover ratio; KPI: key performance indicators; LTE: long-term evolution; MLB: mobility load balancing; NGMN: Next Generation Mobile Networks; OPEX: operational expenditure; OR: outage ratio; PLS: power load sharing; PLUS: power load and user sharing; PRB: physical resource block; PTS: power traffic sharing; PUS: power user sharing; QoS: quality of service; RAT: radio access technologies; RRM: radio resource management; SINR: signal-to-interference-plus-noise; SON: self-organizing networks; UDR: user dissatisfaction ratio; VoIP: voice over IP.

\section{Competing interests}

The authors declare that they have no competing interests.

\section{Acknowledgements}

This work has been partially funded by the Spanish Ministry of Economy and Competitiveness within the National Plan for Scientific Research, Technological Development and Innovation 2008-2011 and the European Development Fund (ERDF), within the MONOLOC project (IPT-430000-2011-1272). This work has been also partially supported by the Junta de Andalucía (Proyecto de Investigación de Excelencia P12-TIC-2905).

Received: 8 August 2014 Accepted: 20 January 2015

Published online: 15 February 2015

\section{References}

1. 3GPP TS 32.500V11.1.0 (2011-12), Telecommunication management; self-organizing networks (SON); concepts and requirements

2. NGMN requirement document. NGMN top OPE recommendations. Sept 2010.

3. 3GPP TR 36.902V9.3.1 (2011-03), Evolved Universal Terrestrial Radio Access Network (E-UTRAN); self-configuring and self-optimizing network (SON) use cases and solutions (Release 9)

4. J Ramiro, K Hamied, Self-Organizing Networks: Self-Planning, Self-Optimization and Self-Healing for GSM, UMTS and LTE (John Wiley \& Sons, New York, 2011)

5. JG Andrews, H Claussen, M Dohler, S Rangan, MC Reed, Femtocells: past, present, and future. IEEE J. Sel. Areas Commun. 30(3), 497-508 (2012)

6. H Claussen, LTW Ho, LG Samuel, Self-optimization of coverage for femtocell deployments. in Wireless Telecommunications Symposium, 2008. IEEE WTS 2008, Pomona, 24-26 April 2008, pp. 278-285

7. H Zhang, X Wen, B Wang, W Zheng, Y Sun, A novel handover mechanism between femtocell and macrocell for LTE based networks, in Second International Conference on Communication Software and Networks, 2010 (ICCSN '10, Singapore, 2010), pp. 228-231

8. D Lopez-Perez, A Valcarce, G de la Roche, J Zhang, OFDMA femtocells: a roadmap on interference avoidance. IEEE Commun. Mag. 47(9), 41-48 (2009)

9. Z Becvar, P Mach, Adaptive hysteresis margin for handover in femtocell networks. in 2010 6th International Conference on Wireless and Mobile Communications (ICWMC) (Valencia, 20-25 Sept 2010), pp. 256-261

10. D Xenakis, N Passas, L Merakos, C Verikoukis, Mobility management for femtocells in LTE-advanced: key aspects and survey of handover decision algorithms. IEEE Communications Surveys \& Tutorials 16(1), 64-91 (2014)
11. A Lobinger, S Stefanski, T Jansen, I Balan, Load balancing in downlink LTE self-optimizing networks. in 2010 IEEE 71st Vehicular Technology Conference (VTC 2010-Spring), (Taipei, 16-19 May 2010), pp. 1-5

12. $P$ Muñoz, D Laselva, R Barco, $P$ Mogensen, Adjustment of mobility parameters for traffic steering in multi-RAT multi-layer wireless networks. EURASIP J. Wirel. Commun. Netw. 2013, 133 (2013)

13. Y Qiaoyang, R Beiyu, C Yudong, M Al-Shalash, C Caramanis, JG Andrews, User association for load balancing in heterogeneous cellular networks. IEEE Trans. Wirel. Commun. 12(6), 2706-2716 (2013)

14. R Kwan, R Arnott, R Paterson, R Trivisonno, M Kubota, On mobility load balancing for LTE systems. in 2010 IEEE 72nd Vehicular Technology Conference Fall (VTC 2010-Fall), (Ottawa, 6-9 Sept 2010), pp. 1-5

15. S Donghoon, C Sunghee, Dynamic power control for balanced data traffic with coverage in femtocell networks. in 2012 8th International Wireless Communications and Mobile Computing Conference (IWCMC), (Cyprus, 27-31 Aug 2012), pp. 648-653

16. JM Ruiz-Avilés, S Luna-Ramírez, M Toril, F Ruiz, I de la Bandera-Cascales, P Munoz-Luengo, Analysis of load sharing techniques in enterprise LTE femtocells. in Wireless Advanced (WiAd), 2011, (London, June 2011), pp. 195-200

17. JM Ruiz-Avilés, S Luna-Ramírez, M Toril et al., Traffic steering by self-tuning controllers in enterprise LTE femtocells. EURASIP J. Wirel. Commun. Netw. 2012, 337 (2012)

18. P Munoz, R Barco, JM Ruiz-Aviles, I de la Bandera, A Aguilar, Fuzzy rule-based reinforcement learning for load balancing techniques in enterprise LTE femtocells. IEEE Trans. Veh. Technol. 62(5), 1962-1973 (2013)

19. H Zhang, X Qiu, L Meng, X Zhang, Design of distributed and autonomic load balancing for self-organization LTE. in 2010 IEEE 72nd Vehicular Technology Conference Fall (VTC 2010-Fall), (Ottawa, 6-9 Sept 2010), pp. 1-5

20. H Zhang, X Wen, B Wang, W Zheng, Y Sun, A novel handover mechanism between femtocell and macrocell for LTE based networks. in Second International Conference on Communication Software and Networks, 2010. ICCSN '10, (Washington DC, 26-28 Feb 2010), pp. 228-231

21. M Toril, $V$ Wille, Optimization of handover parameters for traffic sharing in GERAN. Wirel. Pers. Commun. 47(3), 315-336 (2008)

22. M Sugeno, An introductory survey of fuzzy control. Inform. Sci. 36(1), 59-83 (1985)

23. T Takagi, M Sugeno, Fuzzy identification of systems and its applications to modeling and control. IEEE Transactions on Systems, Man and Cybernetics SMC-15(1), 116-132 (1985)

24. International Electrotechnical Commission (IEC), Technical committee no. 65: industrial process measurement and control. Sub-committee 65 B: devices. IEC 1131 - programmable controllers. Part 7 - fuzzy control programming. Committee draft cd 1.0 (rel. 19 Jan 97)

25. Alcatel Lucent 9362 Enterprise Cell v2.2 Datasheet. http://resources.alcatel-lucent com/?cid=156644. Accessed 1 Feb 2015

26. JM Ruiz-Avilés, S Luna-Ramírez, M Toril, F Ruiz, I de la Bandera, P Muñoz, R Barco, P Lázaro, V Buenestado, Design of a computationally efficient dynamic system-level simulator for enterprise LTE femtocell scenarios. Journal of Electrical and Computer Engineering 2012, 14 (2012)

27. P Kyösti, J Meinilä, L Hentilä, X Zhao, T Jämsä, M Narandzić, M Milojević, C Schneider, A Hong, J Ylitalo, V-M Holappa, M Alatossava, R Bultitude, Y de Jong, T Rautiainen, IST-4-027756 WINNER II D1.1.2. WINNER II channel models. Part II. Radio channel measurement and analysis results. v1.0. Tech. rep. WINNER II IST project 2007

28. T Sorensen, P Mogensen, F Frederiksen, Extension of the ITU channel models for wideband (OFDM) systems, in 2005 IEEE 62nd Vehicular Technology Conference (VTC), vol. 1 (VTC-2005-Fall, Dallas, 2005), pp. 392-396

29. DB Johnson, DA Maltz, Dynamic source routing in ad hoc wireless networks. in Mobile Computing. The Kluwer International Series in Engineering and Computer Science, vol. 353. (Springer US, 1996), p. 153 